\title{
L'éducation à la citoyenneté à la recherche de présences effectives
}

\section{François Audigier}

Tout projet et toute réflexion sur l'éducation à la citoyenneté doivent faire place aux nombreuses tensions et contradictions qui la caractérisent. Une première partie, appuyée sur quelques résultats extraits d'une enquête auprès d'enseignants français, interroge les conceptions de ces enseignants et les modes de présence de cette éducation à l'école primaire. L'auteur situe ces analyses au regard de quelques évolutions importantes de l'école et de la société et des débats correspondants. L'éducation à la citoyenneté demande une réflexion rigoureuse sur ses références théoriques et pratiques, sur les compétences citoyennes, sur les conditions de son existence. Cela conduit à définir trois modes de présence, modes indispensables et complémentaires, pour que l'éducation à la citoyenneté soit à la hauteur des attentes et des ambitions.

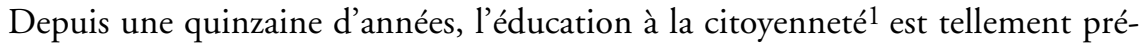
sente dans les propos et intentions sur l'Ecole qu'on pourrait la croire durablement et solidement installée. De nombreux indices incitent à mettre en doute cette présence effective, plus encore à interroger ses modes de présence. Après tout, de si nombreux et de si pressants appels ne cachent-ils pas un certain désarroi ou certains désaccords quant à son contenu, ou encore certaines difficultés à la faire exister dans le quotidien des classes et des établissements? En fait, l'éducation à la citoyenneté est le domaine qui porte à l'extrême de nombreuses tensions et contradictions de l'univers scolaire et des relations que le reste de la société entretient avec lui: il ne saurait y avoir d'éducation à la citoyenneté sans construction de connaissances mais ce qui est important ce sont in fine les comportements, les pratiques, aujourd'hui et demain; l'éducation à la citoyenneté est porteuse de valeurs et à ce titre elle comporte une part de transmission et d'inculcation mais le choix des valeurs relève d'abord de la liberté du sujet; l'éducation à la citoyenneté est un moyen pour intégrer les jeunes générations à la société mais chacun est libre de ses appartenances; l'éducation à la citoyenneté est d'abord construction d'une personne autonome et d'un citoyen libre et critique mais il est important que chacun se sente responsable et solidaire ... L'éducation 
à la citoyenneté est plus qu'aucun autre domaine portée par l'idée de culture commune, d'élaboration de références partagées pour affirmer et faire vivre un espace public de délibération où les citoyens sont égaux, mais cela s'inscrit dans une Ecole qui, aussi voire surtout, une fois les premières années passées, classe, oriente, hiérarchise, élimine, reproduit, promeut, valorise, différencie ...

Ces tensions et contradictions s'expriment diversement selon les Etats, les systèmes éducatifs, les cultures politiques, les conceptions de la citoyenneté. Toutefois, au-delà de ces différences, l'observateur attentif est frappé par la convergence des interrogations, des orientations proposées et des solutions mises en œuvre ${ }^{2}$. L'éducation à la citoyenneté est l'objet de deux interpellations majeures: sa conception et ses modes de présence. La première appelle une réflexion rigoureuse sur les références nécessaires pour la penser et la construire théoriquement et pratiquement. La seconde invite à mettre à distance un discours trop répandu qui voudrait que cette éducation relève presque uniquement de l'expérience et que, à ce titre, elle se réduirait à un ensemble de dispositifs pratiques et à sa diffusion dans la plupart des matières scolaires. Les résultats d'une enquête menée auprès d'enseignants de l'école primaire en France traduisent les hésitations et ambiguïtés dont cette éducation est l'objet. En situant ces tendances au regard, d'une part des évolutions de nos conceptions de la citoyenneté, d'autre part des tensions qui sont au cœur de son existence, l'article plaide pour que, dans nos institutions scolaires, cette éducation soit présente de trois manières nécessaires et complémentaires. Principalement appuyé sur des travaux et des exemples qui concernent l'école primaire, le point de vue présenté ici est aisément transposable à l'école secondaire.

\section{Hésitations et ambiguites: une enquête auprès des enseignants de l'école primaire en France}

Durant l'année 97-98, au cours d'une recherche sur l'état de l'enseignement de l'histoire, de la géographie et de l'éducation civique à l'école primaire en France, ${ }^{3}$ 46 enseignants ont été interrogés lors d'entretiens semi-directifs et 720 par questionnaire. Dans les entretiens comme dans les questionnaires, l'éducation civique est prioritairement associée aux règles de vie en classe, plus largement dans la société. Les secondes sont conçues comme le prolongement des premières. Cette priorité est liée à l'urgence du quotidien. Assurer un minimum d'ordre scolaire est nécessaire. Donner à ce souci une dimension éducative vient dans un second temps. Ainsi, les valeurs sont un domaine délicat et les enseignants se partagent sur l'association de l'éducation civique et de leur transmission. Toutefois, deux valeurs s'affirment, comme des évidences qui ne sont guère interrogées: le respect, respect des personnes et des biens, et la tolérance, avec les ambiguïtés de ce terme entre ouverture et indifférence. La classe est aisément pensée comme une métaphore de la société. Ce qui est valide en classe est valide à l'extérieur de 
la classe; les comportements appris pour l'une devraient être observés ailleurs. Cette généralité laisse place au doute lorsque l'on interroge les enseignants sur les règles de vie hors de la classe mais dans l'école. Si chacun est maître dans sa salle, si des règles communes, toujours présentées comme évidentes, régissent les comportements dans les couloirs ou pendant la récréation, les enseignants reconnaissent très simplement que ce sont les adultes présents ou responsables pendant ces moments-là qui imposent leur loi et surtout, qu'il n'y a guère d'accord ou de concertation entre les adultes sur les manières d'utiliser les règles, encore moins sur les sanctions éventuelles. Le métier d'enseignant est d'abord vécu dans les quatre murs de la salle de classe et de façon solitaire.

La dimension politique de la citoyenneté est souvent renvoyée à plus tard. L'association entre règles de vie et citoyenneté politique se fait autour de cet acte emblématique qu'est le vote. L'accent est alors principalement mis sur les aspects formels, matériels - urne, isoloir, dépouillement, etc. -; les principes sont laissés de côté. Ce renvoi, justifié dans les entretiens par des considérations d'âge des élèves, est d'autant plus intéressant que, dans le même temps, les enseignants interrogés ne font pas, ne font plus?, référence à ce qui constituait théoriquement l'horizon de l'école en France, de l'instruction civique en particulier, à savoir la nation, la république, voire la patrie. Ces mots, ou d'autres qui leur seraient proches, sont absents des entretiens. Cette orientation prioritaire relevée, une proportion non négligeable d'enseignants dit préférer l'étude des institutions politiques, aborder les grands textes des droits de l'homme et avoir quelques doutes sur l'intérêt de travailler la vie en classe. Outre ces deux orientations, l'éducation civique accueille des thèmes variés et nombreux, de la sécurité, routière ou non, à l'étude de l'environnement, de l'introduction de l'actualité à des études locales. Cela lui donne une image très disparate et peu propice à une construction raisonnée et organisée sur la durée.

La priorité accordée aux règles de vie et aux comportements se traduit dans une présence souvent diffuse de l'éducation civique. Moins du tiers des enseignants ayant répondu à l'enquête disent l'enseigner régulièrement, une fois par semaine. En revanche, ils sont plus du tiers à dire le faire de "temps en temps", alors que cette même fréquence n'est indiquée pour l'histoire et la géographie que par $5 \%$ et $7 \%$ des mêmes enseignants. Quant aux liens avec d'autres disciplines, les propos sont très convenus et les exemples précis rares. Les méthodes les plus retenues sont cohérentes avec ces tendances et les renforcent. Faire réfléchir les élèves sur les incidents et sur les règles de vie collective sont des propositions plébiscitées dans le questionnaire. L'image dominante est celle d'une éducation civique prioritairement comportementale et peu orientée vers la construction de connaissances, quels que soient les thèmes étudiés; l'évaluation tient peu de place.

Les réponses au questionnaire ont fait l'objet d'analyses multivariées. L'analyse des correspondances multiples sur les seuls items d'éducation civique organise les réponses des enseignants autour de certaines oppositions et confirme 
ainsi la diversité des conceptions précédemment esquissée. La première opposition sépare d'un côté un ensemble de choix qui privilégie l'étude des institutions politiques, notamment au niveau de la commune, la présence du droit, les relations avec l'histoire et laisse plus de côté ce qui relève du débat, des valeurs, de la morale, des règles de vie, de l'autre des choix favorables aux règles de vie, à la réflexion sur les problèmes de la classe, à la transmission de valeurs, à la morale et des choix négatifs sur les aspects politiques et juridiques, sur l'étude de l'actualité ou la présence de grands textes internationaux. Sur la première orientation, l'analyse montre la présence significative d'hommes, d'enseignants ayant un CM2, dernière classe de l'école primaire en France, aimant l'histoire, sur la seconde, plus de femmes, d'enseignants ayant des élèves plus jeunes, n'aimant pas ou peu l'histoire, pratiquant peu l'écrit avec leurs élèves.

Le second facteur nuance ces oppositions; il met en évidence, d'un côté des relations entre des items qui expriment un avis positif pour l'étude de l'actualité, la recherche de liens entre l'éducation civique et l'histoire et la géographie, les débats, conseils d'élèves, le refus des valeurs et de la morale mais l'accord pour la laïcité et pour une éducation civique facteur d'intégration sociale, de l'autre beaucoup de méfiance pour ce qui est actualité, les journaux, les demandes des élèves, plus d'attrait pour l'usage de fiches et l'étude des institutions politiques et des services publics. Les variables descriptives des populations traduisent une opposition d'âge et d'ancienneté dans le métier avec les plus jeunes pour le premier côté, une opposition de formation, ceux sans formation universitaire plutôt du second côté, et un attrait différent pour l'histoire et l'éducation civique, manifeste chez les premiers, négatif chez les seconds.

A partir de ces analyses, des classifications ont été construites autour de quatre groupes d'enseignants, qui reprennent à leur manière ces oppositions:

- le premier groupe rassemble près d'un tiers des enseignants ayant répondu à l'enquête, un peu plus de femmes et d'enseignants ayant des élèves jeunes. Il se caractérise par un intérêt plus affirmé pour les règles de vie et ce qui concerne la classe, les valeurs, la morale. Ces enseignants sont moins sensibles à tout ce qui est 'extérieur';

- le second groupe réunit la même proportion de personnes. Plus souvent diplômés universitaires, plus jeunes et plus formés dans les IUFM (Instituts universitaires de formation des maitres), ces enseignants, qui plus que les autres aiment l'éducation civique, portent leur intérêt pour l'actualité, la compréhension de la société, les méthodes actives, tous les items qui expriment une ouverture vers l'extérieur;

- le troisième groupe rassemble le quart des personnes interrogées. Les opinions qu'ils expriment sont prudentes; ils doutent plus souvent de l'efficacité du travail sur les règles de vie, ils sont donc moins nombreux à pratiquer les conseils divers ou à travailler ces règles; ils sont un peu plus intéressés par l'actualité, les enquêtes, les recherches, mais doutent de la facilité d'accès aux journaux ou de l'étude des droits des enfants. Ce groupe comporte plus d'en- 
seignants n'ayant pas de formation universitaire et ayant des élèves de CM2;

- le petit sixième restant constitue un dernier groupe plus masculin et enseignant lui aussi plus souvent en CM2. Ils choisissent fortement l'étude des institutions politiques, les concepts juridiques et l'étude des grands textes et moins voire, beaucoup moins, ce qui est règles de vie, valeurs, débats, ce qui est lié à la vie de la classe. Ils tiennent le monde à distance et insistent sur les institutions politiques.

Ces différentes classes illustrent la diversité des conceptions et des modes de présence de l'éducation civique. Elles mettent aussi en évidence les nombreux facteurs qui interviennent dans les choix faits par les enseignants. La première remarque, presque paradoxale dans un système réputé centralisé comme le système français, est la quasi absence de références aux programmes officiels au cours des entretiens; cette absence est aussi remarquable dans les choix exprimés dans les réponses au questionnaire. La seconde remarque observe la combinaison complexe des différents facteurs - âge des enseignants, âge des élèves, formation, appréciation sur le milieu social des élèves, attitudes par rapport à la discipline -. Les enseignants qui pensent l'éducation civique constamment présente puisqu'elle privilégie les comportements et les attitudes, ne l'accompagnent pas de l'identification et de l'enseignement de contenus précisément reconnus. Une fois le moment de définition ou de travail sur les règles en début d'année, le rappel semble l'usage le plus fréquent de ces règles. Seules les structures de conseil donnent un peu plus d'assise à ces pratiques. Cette tendance forte énoncée, l'éducation civique est un grand bazar, à l'image du monde?, dans lequel chacun place au gré des circonstances, de ses intérêts et de ceux de ses élèves, des thèmes amples, plus ou moins reliés à l'histoire, une sensibilité pour l'actualité, des travaux liés à l'environnement local et bien d'autres choses. Tout ceci, principalement destiné à «faire prendre conscience [...]» espérant ainsi développer des curiosités et des comportements d'adultes mieux informés. Il ne nous appartient pas de porter un jugement sur cette diversité des conceptions et des pratiques, des contenus et des approches, telle que l'expriment les enseignants interrogés. Si celle-ci traduit une réalité, elle est inapte à nous aider dans la construction d'un projet d'éducation à la citoyenneté qui prenne au sérieux l'idée même de citoyenneté et la préparation des jeunes générations à exercer les droits et obligations de citoyen dans une société démocratique.

\section{Trois nécessites Pour construire un projet d'éducation à la citoyennete: évolutions, tensions et références}

L'interprétation des conceptions et des hésitations que cette enquête exprime, requiert plusieurs niveaux d'analyse. Réfléchir sur l'éducation à la citoyenneté et la mettre en œuvre impliquent de refuser de s'enfermer dans le quotidien des 
classes et des établissements, dans les limites étroites de tel ou tel institution ou système politique. La citoyenneté et les interrogations dont elle est aujourd'hui l'objet, les incertitudes, difficultés, hésitations et espoirs que nos sociétés produisent et affrontent, interdisent tout rétrécissement. Après tout, nous ne savons pas grand chose du monde que les élèves auront en charge, au cours de leur existence d'adultes d'ici 10 ou 20 ans, voire 40 ou 50 ans. Cela exige de la part des enseignants et des responsables des institutions éducatives, modestie et ambition, rigueur et ouverture. Pour construire et mettre en ouvre un projet d'éducation à la citoyenneté, trois ensembles de données sont à prendre en considération: les évolutions dont nos conceptions de la citoyenneté sont l'objet, les tensions qui traversent obligatoirement cette éducation, les références théoriques et pratiques avec lesquelles la penser et la conduire.

\section{Nos conceptions de la citoyenneté ont changé}

La première évolution a trait aux ambiguïtés du concept de citoyenneté et à ce qui est souvent énoncé comme un recul du politique. Après une période où la connaissance des institutions politiques était un ancrage privilégié de l'éducation à la citoyenneté, l'accent est fortement mis aujourd'hui sur la participation, l'autonomie de la personne, la société civile. ${ }^{4}$ Cette mise à distance est aussi accentuée par la présence d'élèves d'origine étrangère dans de nombreuses écoles. En effet, la citoyenneté politique juridiquement associée à la nationalité concerne de façon plus distante ces élèves. Par exemple, Payet (1997) distingue «deux formes de citoyenneté [...]. Plutôt que de citoyenneté politique, nous parlerons ici de citoyenneté pratique ou ordinaire, et ce dans l'école» (p. 99). Quoi qu'il en soit de ce débat indispensable, le constat général est celui d'un élargissement considérable de ce qui est rangé sous le terme de citoyenneté, et donc par là même des contenus et pratiques d'une éducation à la citoyenneté. La citoyenneté juridique et politique, qui constitue toujours le socle de la citoyenneté démocratique, est très souvent recouverte par la citoyenneté sociale, la citoyenneté économique voire la citoyenneté culturelle. ${ }^{5}$ Le risque, pour l'école, est de considérer que, puisque tout acte social a une dimension citoyenne, l'éducation à la citoyenneté est partout, que, puisque la citoyenneté s'exprime à travers des attitudes et des manières de vivre, elle est d'abord voire uniquement une éducation aux comportements vis à vis des autres, de la collectivité et des institutions. Cette tendance est accentuée par les mises en question dont l'univers du politique est l'objet. ${ }^{6}$ Dans l'éducation, l'abandon ou la mise de côté de cet univers qui accompagnent le sentiment de son déclin, renforcent les tendances à limiter cette éducation à ce qui relève de l'ordre scolaire. Cette réduction est paradoxale, voire inquiétante, lorsque l'on observe le monde d'aujourd'hui dans lequel les autres champs de l'activité humaine, économique ou culturel par exemple, dépassent très largement nos territoires politiques et nous obligent, pour les comprendre et agir, à penser à différentes échelles, du local au mondial, à nous ouvrir aux autres.

Ce déclin du politique et l'élargissement du contenu de la citoyenneté ac- 
compagnent une seconde évolution exprimée ici par l'idée du glissement d'une citoyenneté d'appartenance et d'obéissance vers une citoyenneté instrumentale et d'autonomie. Lorsque, autour de la fin du XIXe siècle, la plupart des systèmes éducatifs européens introduisent de l'instruction civique, l'apprentissage organisé des institutions politiques aux différentes échelles avait pour but premier de développer un sentiment d'appartenance à la communauté politique dans laquelle vit l'enfant; ce sentiment accompagne l'importance accordée à l'obéissance aux lois, au respect de ses devoirs par le citoyen. La priorité est de développer un sentiment d'adhésion à l'égard des communautés politiques modernes qui s'affirment alors. L'affaire est connue et a donné lieu à de nombreuses études. Depuis quelques dizaines d'années, l'appartenance n'est plus vécue ou pensée de façon aussi privilégiée comme appartenance à une communauté politique. Elle fait place de façon explicite, parfois revendiquée, à la pluralité et à la mobilité. La citoyenneté n'est plus alors, plus seulement?, une citoyenneté d'adhésion, mais devient une citoyenneté plus instrumentale. Etre citoyen, c'est être titulaire de droits, droits qu'il convient avant tout de faire valoir par tous les moyens légaux possibles. Il y a une instrumentalisation de la citoyenneté et du droit qui accompagne l'affirmation de l'individu et la priorité accordée à ses libertés, à ses pouvoirs. Certes, on tente bien de rappeler l'importance de la participation, de la solidarité ou de la responsabilité, autant de rappels de la dimension de co-citoyenneté intrinsèque à la réalité citoyenne elle-même.

De plus, la citoyenneté d'appartenance incluait une forte composante affective, elle-même exprimée et constamment ranimée par des actes symboliques tels que les commémorations des faits les plus importants de la communauté d'appartenance. Cette dimension affective, généralement liée à l'histoire, a été à juste titre fortement mise en cause en raison des nombreuses dérives qu'elle a connues, des comportements sectaires voire dramatiques qu'elle a souvent renforcés sinon provoqués. Mais est-il possible de se reconnaître membre d'une communauté politique, quelle que soit son échelle, locale, cantonale ou régionale, nationale, planétaire, sans partager d'une manière ou d'une autre quelque chose qui la situe dans le temps, dans le devenir historique, avec les trois dimensions du passé, du présent et de l'avenir? Il se pourrait que l'individu moderne, sommé de se déterminer librement, d'être en permanence capable de faire la preuve de ses compétences, de choisir ses propres valeurs, d'affirmer son autonomie, ait de plus en plus de difficultés à ne pas se soumettre, de fait, aux pouvoirs qui délimitent les espaces et les objets de sa liberté. ${ }^{7}$

\section{L'éducation à la citoyenneté sous trois tensions}

Les évolutions de nos conceptions de la citoyenneté déstabilisent profondément l'éducation. La citoyenneté, tant les savoirs que les pratiques qui lui sont associées, n'offre donc pas une référence stable pour en construire l'éducation. Une analyse historique plus fouillée montre aussi que cette éducation a toujours été aux prises avec de fortes tensions. Parmi celles-ci, nous en retenons trois qui à 
leur manière sont fondatrices. Il s'agit de les accepter et non de les ignorer, de travailler avec elles et de les travailler avec les élèves.

La première tension s'exprime dans le couple autonomie/héritage. Depuis qu'elle existe dans sa forme moderne, l'Ecole a toujours été écartelée entre une éducation fondée sur la transmission systématique et organisée de savoirs et de savoir-faire, de compétences accumulées par les générations précédentes vers les suivantes, et une éducation fondée sur une interrogation libre et raisonnée de la vie elle-même telle qu'elle se présente à chaque individu, comme expérience vécue. La constitution des sciences, naturelles et sociales, selon les manières dont elles ont été découpées et développées depuis les XVI-XIXe siècles, a accentué l'importance accordée à la première orientation. Celle-ci a conduit la mise en place des filières d'enseignement destinées aux classes dirigeantes, tandis que la seconde a plus largement inspiré les filières destinées à la formation des classes populaires, des travailleurs manuels. Au fondement de cette séparation, outre les données proprement sociologiques et politiques, se place l'opposition entre intellectuel et manuel, théorie et pratique. Cette séparation vole en éclat aujourd'hui. La prolongation de la scolarisation et les demandes de démocratisation mettent en cause les séparations trop étanches entre ces filières; le poids de plus en plus pesant accordé au présent ${ }^{8}$ affaiblit la référence à des connaissances construites progressivement dans des disciplines identifiées et le temps nécessairement long pour entrer dans ces disciplines. Le temps de l'apprentissage est d'autant plus étalé que de nombreux savoirs n'ont pas, en eux-mêmes, une signification immédiate pour une grande majorité des élèves. L'éducation à la citoyenneté se veut à la fois transmission de valeurs, d'un monde déjà là avec ses normes et sa culture, et construction d'un sujet, d'une personne autonome qui, prenant sa place dans ce monde, contribue à son évolution, à sa dynamique.

La seconde tension porte sur la relation entre savoirs et pratiques. L'éducation à la citoyenneté dans sa version traditionnelle était décriée parce que les contenus qu'elle enseignait n'avaient guère de sens pour les élèves. Ceux-ci n’arrivaient pas à établir des relations entre ce qui était enseigné et les expériences qu'ils avaient de la vie scolaire et sociale. Devant le peu de succès de cet enseignement trop formel, la priorité est alors mise sur les pratiques sans que cette référence aux pratiques soit clairement pensée et construite: pratiques sociales, pratiques plus spécifiquement politiques, pratiques citoyennes courantes, pratiques de ceux qui font plus délibérément acte ou profession d'action dans l'univers politique, pratiques de conformité et de respect des lois, pratiques d'engagement, etc. Un double travail de clarification est alors nécessaire, d'une part sur les pratiques elles-mêmes, celles qui sont à placer en référence à l'éducation à la citoyenneté, d'autre part sur les relations entre les savoirs et les pratiques.

En effet, les relations entre savoirs et pratiques se nouent de manière tout à fait particulière dans l'espace scolaire. La troisième tension retenue ici s'énonce autour du couple discipline scolaire/questions de sociétés et prolonge les deux couples précédents. Les savoirs et savoir-faire qu'il convient de construire pen- 
dant la scolarité sont distribués dans des disciplines scolaires, identifiées par des dénominations, des horaires, des curriculums, programmes et autres plans d'étude. Cette distribution inscrit l'enseignement dans une certaine temporalité. Ce qui est enseigné se présente selon un ordre établi qui a peu de choses à voir, a priori, avec les urgences du monde, avec les besoins de connaissances et de compétences que la vie sociale requiert. Par leur construction, les disciplines scolaires impriment une certaine vision du monde et de la connaissance. L'ordre dans lequel sont enseignés leurs contenus spécifiques et la manière dont elles le font ne sont pas directement liés à la vie sociale. Si dans le quotidien des classes, de nombreux enseignants travaillent sur la relation entre les savoirs enseignés et l'expérience des sujets, ce n'est pas celle-ci qui commande la construction des curriculums et plans d'étude.

Depuis plusieurs décennies, on demande à l'Ecole d'introduire des éducations, éducations aux médias, à la santé, à l'environnement, à la paix, à la prévention des risques, etc. Le terme d'éducation désigne clairement ici le but pratique. Eduquer à la santé, c'est pour que les jeunes résistent au tabac et aux drogues, utilisent des préservatifs pour se prémunir des maladies sexuellement transmissibles, se nourrissent correctement pour éviter l'obésité ... La croyance sur laquelle est fondée l'école postule que plus de connaissances induit des comportements plus rationnels, mais trop d'expériences et de situations mettent en doute cette croyance. Le dilemme scolaire est alors total: faut-il inscrire ces préoccupations dans des disciplines constituées, les "disciplinariser», les soumettre aux logiques curriculaires de l'enseignement de la biologie, de celui de telle ou telle science sociale, en inventer de nouvelles? Ou convient-il de choisir quelques thèmes, plus proches de la manière dont les questions se posent dans la vie quotidienne et à partir de là, sur cette base, convoquer divers savoirs pour informer ces questions? Accorder la priorité à la première manière c'est prendre le risque que les savoirs correspondants, enfermés dans les logiques disciplinaires n'arrivent pas à prendre de sens par rapport aux pratiques, restent trop extérieurs au sujet; aborder ces questions comme autant de questions que la société se pose, c'est prendre un autre risque, celui de sombrer dans des pratiques informelles, de se laisser porter au gré des urgences voire des modes. Ce second risque est aussi d'empêcher les élèves d'entrer dans les constructions raisonnées que sont les disciplines scolaires, dans ce qu'elles portent d'initiation et d'ouverture à des savoirs rigoureux, patiemment construits par l'humanité.

\section{De la rigueur sur les références}

Tout projet d'éducation et tout enseignement sont construits en référence à des données qui les unes leur sont extérieures, les autres correspondent aux modes de fonctionnement de l'école. Si les finalités commandent les choix de ce qui est enseigné et les dispositifs de vie scolaire mis en place dans les établissements, ces choix et ces dispositifs empruntent savoirs, savoir-faire, pratiques, attitudes, etc., à des domaines de référence pour ensuite les recomposer selon les logiques propres 
de l'école. Lidentification des références de l'éducation à la citoyenneté est particulièrement difficile et délicate. Nous n'avons aucunement affaire ici à un corpus stable ni dans le monde social, ni dans le monde scolaire. Une tentation est alors de laisser chaque établissement, voire chaque enseignant se débrouiller; après tout, nous sommes tous citoyens et donc tous compétents en matière de citoyenneté. Point n'est besoin d'énoncer les risques d'une telle dérive. L'autonomie des écoles et des enseignants appelle encore plus de rigueur, de capacités réflexives et de compétences. Un travail sur les références théoriques et pratiques de la citoyenneté et sur les compétences à construire est plus que jamais requis.

Ainsi, la citoyenneté porte en elle aujourd'hui des exigences de compréhension du monde et de présence active à ce même monde qui obligent à lui donner un sens large et dynamique. Un projet d'éducation à la citoyenneté mobilise diverses références. Les premières tiennent à la définition des compétences que l'on attend d'un citoyen, compétences elles-mêmes dépendantes de la conception de la citoyenneté en vigueur dans une communauté politique. Les secondes relèvent des théories de l'apprentissage, de la construction de connaissances et de représentations sur la société, les valeurs et les normes, le vivre ensemble. Un projet dynamique d'éducation à la citoyenneté se construit sur ces deux ensembles de références, avec leurs incertitudes et leurs ouvertures. Nous n'ouvrons ici la réflexion que sur le premier ensemble. L'accord est aujourd'hui unanime dans l'abondante littérature qui lui est consacrée, les compétences du citoyen dépassent largement la seule maitrise de quelques connaissances sur les institutions; elles sont très largement du domaine de l'action. Mais cet accent mis sur l'action et le vivre ensemble ne doit pas conduire à négliger les connaissances. La mission et la raison d'être de l'école dans une société démocratique sont de mettre les élèves en situation de construire des outils théoriques et pratiques pour penser leur vie sociale et agir.

Ce sont ainsi plusieurs impératifs qui se combinent. S'efforçant de construire des compétences sociales, l'éducation à la citoyenneté implique des activités et des travaux du côté des expériences que les élèves ont de la vie sociale, de la vie scolaire notamment. Les compétences citoyennes emportent également avec elles des connaissances sur le monde et des capacités pour participer au débat public; cette éducation requiert donc l'étude de différents aspects du monde social, lesquels ne se rapportent ni de façon exclusive ni de façon systématique à une seule discipline scolaire. Enfin, dans une société démocratique, le citoyen est une personne titulaire de droits et d'obligations et, avec les co-citoyens, de la souveraineté politique; l'éducation à la citoyenneté demande donc d'introduire les élèves à l'univers juridique, à travers la construction de quelques concepts clés et l'étude des pratiques et des dispositifs conformes au droit démocratique; elle demande aussi d'expliciter et de réfléchir les principes et les valeurs qui fondent le droit et la souveraineté, y compris dans leurs dimensions problématiques et conflictuelles. ${ }^{9}$ Enfin, l'éducation à la citoyenneté est une éducation au pouvoir. 


\section{Trois modes de présence pour l'éducation à La citoyenneté:}

Pour opérationnaliser ces orientations, trois modes de présences s'imposent: dans et avec la vie scolaire, le fonctionnement des établissements et des relations entre les personnes; une initiation aux problèmes de société; des moments clairement identifiés consacrés à la construction de certaines compétences spécifiques. ${ }^{10}$ Ces trois modes sont complémentaires; il ne s'agit pas de choisir l'un ou détriment de l'autre; ils doivent être liés de manière explicite. Chacun de ces modes fait l'objet d'une brève présentation avec un exemple plus précis pour ce qui concerne la vie scolaire.

\section{La vie scolaire, l'exemple du travail sur les règles de vie dans la classe}

L'éducation à la citoyenneté a deux conséquences majeures pour la vie scolaire. La première est d'ordre simplement logique: si l'école prétend transmettre un certain nombre de valeurs et de principes jugés fondateurs de la vie en commun, son fonctionnement ne doit pas être en contradiction avec ces valeurs et ces principes. La seconde découle de sa fonction d'éducation et d'instruction: l'école doit faire de la vie scolaire un moment d'expérience et de travail pour construire des compétences citoyennes. De là découle l'intérêt de tous les dispositifs de participation, de médiation, de régulation des contenus, tout en rappelant la référence nécessaire aux contenus de la citoyenneté. Ce sont quelques-unes des conséquences de cette référence qui sont énoncées en prenant pour exemple le travail sur les règles de vie.

Sous des dénominations diverses, règles de vie, chartes, règlements, etc., faire énoncer par les élèves les règles qui organisent la vie dans la classe est devenu, comme en témoigne l'enquête présentée dans la première partie, un lieu commun pédagogique à l'école primaire. De nombreux dispositifs décrits dans les ouvrages traitant de cet objet ${ }^{11}$ convergent vers l'idée d'une énonciation qui serait effectuée par les élèves; les règles adoptées surgissent d'une sorte d'accord, d'un sens commun partagé par le groupe de 20 ou 25 élèves sous la conduite plus ou moins vigilante de l'enseignant. On assiste ainsi à une auto-énonciation de ces règles.

Si l'intention générale n'est pas contestable, la référence à la citoyenneté et à la démocratie invite à un examen critique de ces pratiques. Ainsi, par exemple, la démocratie n'est pas un système d'auto-institutionnalisation permanente des règles. Il est dès lors important de ne pas faire croire aux élèves qu'ils ont le pouvoir d'instituer la loi et que les idées qu'ils expriment sont leurs créations originales. Toute règle particulière est toujours liée à d'autres textes qui eux-mêmes reposent sur des valeurs et des principes qui leur sont antérieurs, supérieurs et extérieurs. Il convient aussi de distinguer ce qui relève des nécessités de toute collectivité, de la mise en œuvre du droit à l'éducation, des droits de la personne, 
etc. Mettre la citoyenneté comme principe organisateur de telles pratiques demande de déplacer les objets de travail. A titre d'exemple, voici quatre objets qui concernent l'usage de ces règles et la question du pouvoir:

- la classe ne saurait être à la fois le pouvoir législatif qui fait les lois, le pouvoir exécutif qui met en œuvre leur usage, le pouvoir judiciaire qui décide en cas de manquement;

- si les règles de vie dans une classe ne doivent pas être en contradiction avec celles qui ont cours dans l'ensemble de l'établissement, il est important que les adultes soient d'accord entre eux et que les usages qu'ils font des règles, quels que soient les lieux et les modes de leur énonciation, soient cohérents;

- tant que les relations sont pacifiques, il n'y a guère de problèmes. Les difficultés surgissent lorsque la violence perturbe les relations humaines et l'ordre scolaire. Les conflits violents au sein de l'école sont alors une occasion de travail et de réflexion avec les élèves. Ces moments de réflexion sont distincts des moments de la décision, moments où une autorité s'affirme et s'assume. Elèves et adultes, enseignants et autres, ne sont pas égaux. Egaux en droits, devant bénéficier des mêmes droits fondamentaux de la personne et des mêmes protections, oui, mais inégaux en responsabilités et en pouvoirs liés à leur fonction;

- le dernier exemple insiste sur la question du pouvoir. Les droits sont des libertés et des pouvoirs. Toute éducation à la citoyenneté est une éducation aux pouvoirs. Quels pouvoirs les adultes, aux différents niveaux et en différents lieux et moments de l'institution et de la vie scolaire, sont-ils prêts et disposés à laisser aux élèves?

\section{Les collaborations entre disciplines}

Nous l'avons écrit, les problèmes sur lesquels le citoyen doit se prononcer et dont il est invité à débattre ne sont pas disciplinaires; les décisions prises à leur sujet ne relèvent pas d'un choix scientifique mais d'un choix politique. De plus, si tout citoyen est en principe invité à participer au débat public, nul n’a le désir, l'intérêt ou les dispositions pour intervenir constamment sur n'importe quel objet. Non disciplinaires, reproduisant et exprimant le foisonnement de nos sociétés, ces problèmes ne fournissent aucun repère pour construire des curriculums. D’un autre côté, les références avec lesquelles construire et résoudre ces problèmes, notamment les références scientifiques, sont plurielles et dispersées; elles offrent une pluralité de problématiques et de points de vue. Quelles que soient les difficultés théoriques et pratiques rencontrées, l'étude de ces problèmes impose une collaboration entre les disciplines scolaires concernées.

\section{Un temps spécifique nécessaire}

Que cela constitue ou non une discipline au sens habituel du terme, le troisième mode de présence de l'éducation à la citoyenneté est celui d'un temps spécifique. Il y a des savoirs, des savoir-faire et des compétences propres à la citoyenneté qui, 
sauf exception, ne sont pas pris en compte dans les autres matières scolaires. Deux objets et une orientation sont ici privilégiés: les concepts, les valeurs, et les relations entre concepts, valeurs et expériences de la vie scolaire et sociale. Les concepts sont en premier lieu les concepts juridiques. Les valeurs sont celles des droits de l'homme, fondatrices des systèmes politiques démocratiques. Rien ne sert de proclamer l'autonomie du sujet et les libertés dont il est titulaire s'il n'est pas aussi introduit, de façon rigoureuse et critique, dans ce monde de valeurs et de principes qui lui préexiste. L'autonomie comme la liberté ne sont pas des états mais un mouvement. Les valeurs liées à la citoyenneté démocratique accompagnent ce mouvement. Systématique et réflexif, ce travail demande du temps, le temps de la formation scolaire et au-delà; il ne peut être noyé dans un méli-mélo comportemental.

\section{Conclusion}

Partis des résultats d'une enquête auprès d'enseignants du primaire en France, nous avons mis en évidence les tensions et contradictions propres à l'éducation civique et la diversité de ses modes de présence. Nous avons ensuite argumenté sur une double nécessité: une réflexion ouverte qui prenne en compte les contextes politiques, économiques, sociaux et culturels dans lesquels se déploient cette éducation et donc le refus d'une éducation trop strictement comportementale et trop enfermée dans l'expérience immédiate; une référence rigoureuse aux contenus et aux pratiques de la citoyenneté, quelles que soient les interrogations dont celle-ci est l'objet. Cette référence est la seule garantie pour que se déploie une éducation à la citoyenneté digne de son nom. Toute société a ses règles et ses coutumes, ses habitudes de vie; les unes et les autres sont transmises de générations en générations. Il n'y a pas besoin de faire référence à la citoyenneté pour ne pas taper sur son voisin, traverser dans les clous, ne pas injurier les enseignants ou ne pas bousculer la vieille dame. En appeler à la citoyenneté place l'éducation scolaire face à des exigences et des valeurs particulières. Les préoccupations liées à l'ordre scolaire, à la pacification des relations sociales, aux civilités comme il est souvent dit aujourd'hui, sont légitimes; il est nécessaire d'y répondre. Y répondre en référence à la citoyenneté implique quelques conséquences dont nous avons esquissé les grands axes.

Enfin, il y a quelque danger à faire croire aux élèves que les principes et les valeurs mis en avant et enseignés sont ceux qui inspirent constamment la société, notamment les adultes. Les élèves remarquent vite les distances qui existent entre ces valeurs, ces principes et la vie quotidienne. Cette distance est une exigence supplémentaire pour les adultes, puisque, à l'école, ce sont eux qui sont dépositaires de l'autorité. Il leur revient d'assumer leur autorité dans le respect des droits de chacun, notamment des élèves. L'éducation à la citoyenneté place la barre très haut. 


\section{Notes}

1 Dans cet article, sauf précision argumentée, nous employons le terme d'éducation à la citoyenneté pour désigner cette préoccupation ou ce domaine de manière générale, et le terme d'éducation civique lorsqu'il s'agit de la discipline scolaire avec un horaire et un programme. C'est notamment le terme employé en France.

2 Voir, par exemple, les publications du Conseil de l'Europe autour du projet Education à la citoyenneté démocratique, 1998-2000, disponibles sur le site du Conseil (http://www.coe.fr/).

3 Recherche collective à laquelle participaient des équipes de 12 Instituts universitaires de formation des maîtres (IUFM) et dirigée, dans sa phase initiale, par l'auteur de l'article alors en poste à l'Institut National de Recherche Pédagogique à Paris (Audigier \& TutiauxGuillon, 2003). Sans être strictement représentatif, mais sur quels critères?, l'échantillon interrogé selon les deux méthodes a une composition suffisamment variée pour autoriser quelques généralisations. Voir également Audigier (1999a).

4 Ainsi aux Etats-Unis, la socio-civic education a remplacé la civic education (voir Gross \& Dynneson, 1991).

5 Voir, par exemple Schnapper \& Bachelier (2000).

6 Par exemple, Revault d'Allones (1999).

7 Par exemple, D’Iribarne (1996).

8 Par exemple, Laïdi (2000).

9 Nous avons proposé une esquisse de référentiel de compétences pour l'éducation à la citoyenneté dans une étude pour le Conseil de l'Europe (Audigier, 2000b).

10 Voir par exemple, Audigier (1999a ,2000a , 2001).

11 Par exemple, Leleux (2000).

\section{Références bibliographiques}

Audigier, F. (1999a). L'éducation à la citoyenneté. Paris: INRP.

Audigier F. (1999b). L'éducation civique à l'école primaire et au collège en France: un ancien projet, des tensions permanentes, une actualité renouvelée. CIFEDHOP Collection Thématiques, 7, 33-51.

Audigier, F. (2000a). L'éducation à la citoyenneté, deux particularités: un domaine instable, des références problématiques. In M. Nicolet \& D. Rouyet (Ed.), Education aux citoyennetés. Actes du colloque romand 1998-1999 (pp. 101-112). Neuchâtel: secrétariat général de la Conférence intercantonale de l'instruction publique de la Suisse romande et du Tessin (CIIP).

Audigier, F. (2000b). Concepts de base et compétences clés pour une éducation à la citoyenneté démocratique. Strasbourg: Conseil de l'Europe.

Audigier, F. (2001). Les contenus d'enseignement plus que jamais en question. In C. Gohier \& S. Laurin (Ed.), La formation fondamentale, un espace à redéfinir (pp. 141-192). Outremont: Editions Logiques.

Audigier, F. \& Tutiaux-Guillon, N. (Ed.) (2003). Regards sur l'éducation civique, la géographie et l'histoire à l'école élémentaire. Paris: INRP.

D’Iribarne, P. (1996). Vous serez tous des maîtres. Paris: Seuil.

Gross, R. \& Dynneson, (1991). Social Perspectives on Citizenship Education. Teachers College, Columbia University.

Laïdi, Z. (2000). Le sacre du présent. Paris: Flammarion.

Leleux, C. (2000). Education à la citoyenneté. Bruxelles: De Boeck.

Payet, J.-P. (1997). L'école et la construction de la citoyenneté. In A. van Zanten (Ed.) La scolarisation dans les milieux «difficiles» (pp. 99-113). Paris: INRP.

Revault d'Allones, M. (1999). Le dépérissement de la politique. Paris: Aubier.

Schnapper, D. \& Bachelier, C. (2000). Qu'est-ce que la citoyenneté? Paris: Gallimard. 


\section{Politische Bildung: Auf der Suche nach den Orten ihrer Verwirklichung}

\section{Zusammenfassung}

Jedes Projekt zur und jede Reflexion über die politische Bildung müssen die zahlreichen Tendenzen und Widersprüche, die diese charakterisieren, berükksichtigen. In einem ersten Teil wird, gestützt auf einige ausgewählte Resultate aus einer Befragung französischer Lehrpersonen, nach den Konzeptionen dieser Lehrpersonen und der Art und Weise der Präsenz der politischen Bildung in der Primarschule gefragt. Der Autor situiert diese Analysen im Hinblick auf einige wichtige Entwicklungen der Schule, der Gesellschaft und der darauf bezogenen Debatten. Die politische Bildung verlangt eine rigorose Reflexion über ihre theoretischen und praktischen Referenzpunkte sowie über die angestrebten staatsbürgerlichen Kompetenzen und deren Existenzbedingungen. Dies führt dazu, drei Bedingungen zu definieren, die komplementär vorhanden sein müssen, wenn die politische Bildung den auf sie gerichteten hohen Erwartungen und Ambitionen gerecht werden will.

\section{L'educazione alla cittadinanza ricerca una presenza effettiva}

\section{Riassunto}

Tutti i progetti e tutte le riflessioni attorno all'educazione alla cittadinanza devono fare i conti con numerose tensioni e contraddizioni. In una prima parte del contributo ci si interroga, a partire dai risultati di un'inchiesta svolta in Francia, sulle concezioni degli insegnanti e le modalità di presenza di questo tipo di educazione nella scuola elementare. L'autore mette in rapporto l'analisi con alcune tendenze importanti della scuola, della società e dei relativi dibattiti. L'educazione alla cittadinanza richiede una riflessione rigorosa dei referenti teorici e pratici, delle competenze dei cittadini e delle condizioni della sua esistenza. Su questa basi si arriva a definire tre modalità di presenza, indispensabili e complementari affinché l'educazione alla cittadinanza possa essere all'altezza delle aspettative.

\section{Education citizenship: Where does it comes into realization?}

\footnotetext{
Abstract

Projects on and thoughts about citizen education must make room for tension and contradiction. This is a characterization of the subject. A first part, based upon a few results taken from an investigation of French teachers, questions the teacher's conceptions and the manner of this education at the primary school level. The author situates the analysis in regards to several important evolutions of
} 
school and of the society and of corresponding debates. Citizen education demands rigorous reflection on its theoretical and practical references, on the citizen's competence, on the conditions of its existence. This article is driving at defining three manners/ways of presence, indispensable and complementary manners/ways, in order that citizen education reaches its level of ambition and expectation. 